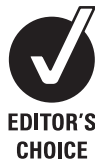

CHOICE

\title{
Ten things your emergency department should consider to prepare for pandemic influenza
}

\author{
S M Robinson, ${ }^{1}$ H R Sutherland, ${ }^{1}$ D J W Spooner, ${ }^{1}$ T J H Bennett, ${ }^{1}$ C-H A Lit, ${ }^{2}$ \\ C A Graham ${ }^{3}$
}

${ }^{1}$ Emergency Department, Addenbrookes Hospital, Cambridge, UK; ${ }^{2}$ A\&E Department, Princess Margaret Hospital, Lai Chi Kok, Kowloon, Hong Kong; ${ }^{3}$ Accident and Emergency Medicine Academic Unit, Chinese University of Hong Kong, Prince of Wales Hospital, Shatin, New Territories, Hong Kong

Correspondence to: Dr S Robinson, Emergency Department, Addenbrookes Hospital, Hills Road, Cambridge CB2 200, UK; susan.robinson@ addenbrookes.nhs.uk

Accepted 18 November 2008

\begin{abstract}
Pandemic influenza remains a potential major threat to global public health. It is essential for emergency departments to be involved in planning for the management of such a major event. It is also important for emergency departments to be clear on their internal arrangements for staff and for patient care. This paper outlines 10 suggestions for UK emergency departments based on the recent experience of emergency departments in Hong Kong and elsewhere.
\end{abstract}

A team from the emergency department of Addenbrookes Hospital, Cambridge recently visited Hong Kong to benefit from the experience of colleagues in emergency medicine and intensive care in dealing with the severe acute respiratory syndrome (SARS) epidemic. Most of these lessons were related to changes in process such as screening and isolation, staff training and managing some of the practical and ethical issues of such an event. However, the most important lesson learnt was that living through an epidemic or pandemic would be one of the most emotive and challenging experiences of our professional lives. The best method of mitigating against this is for emergency departments to ensure they have prepared their staff for such an eventuality to the best of their ability.

The most recent publication from the Department of Health, "Pandemic Flu: Surge Capacity and Prioritisation in Health Services", suggests it will take as little as 4 weeks for pandemic flu to reach the UK once it begins in the country of origin, likely to be in south-east Asia. Once in the UK, its peak could potentially occur only 50 days after entry to the country. This Department of Health document recommends the initiation of training for staff with specific pandemic support or cross-cover roles in the presurge stage (UK level 1).

The immense nature of the task of preparing for a pandemic must not be underestimated. The training and preparation of staff must not be left until a UK level 1 alert is declared; 4 weeks is too short a time to complete this task properly. Very few people have any experience of such an event. Colleagues in Hong Kong have been through the experience of SARS and other infectious disease outbreaks over recent years and have given useful advice based on their experiences.

Forward planning and preparation are key to the successful management of any major incident or outbreak. Core issues to be covered in any preparation include the use of personal protective equipment (PPE) and, in particular, hospital and trust-wide "fit testing". "Fit testing" is the process whereby all staff are tested to find a suitable mask (eg, the UK recommended FFP3 mask or N95 mask in Hong Kong) to suit the specific requirements to have a high-quality seal to protect staff from airborne cross-infection. This needs to be repeated at regular intervals and clearly takes a great deal of time when all trust staff require appropriate masks.

Agreement of standard patient care pathways for use in outbreak conditions should also be secured between departments, and it must be recognised that these pathways may vary depending on the level of the outbreak and its nature.

\section{TEN POINTS TO CONSIDER IN PREPARATION FOR} PANDEMIC INFLUENZA

The 10 points which an emergency department should consider when preparing for pandemic influenza are listed in box 1.

\section{Ensure emergency department representation at the Trust level}

All category 1 responders, as defined in the Civil Contingencies Act, have had to nominate a flu lead; this person has the responsibility of ensuring that planning is taking place. Find out who your local lead is and ask which committee in your hospital is responsible for planning for pandemic flu.

Ensure there is representation from the emergency department. Liaise on issues that require trust involvement such as the procurement and stockpiling of equipment and masks or on the arrangements for fit testing staff outside the emergency department, such as cleaners or medical students. There are significant financial implications associated with planning and training for pandemic flu, and agreement to meet these costs needs to be assured at the highest level.

\section{Talk about pandemic flu with your staff}

Discuss the national plans for preparing and managing pandemic flu and summarise the current guidance from the Department of Health. Inform them of the local arrangements for preparing for flu and how processes within the ED may need to change. Once informed, staff will more readily appreciate the reasons for and importance of fit testing and training in gowning up and gowning down. As a result, compliance and attendance is likely to be better. To do this you will need to read the information available on the Department of 
Box 1 Ten points to consider in preparation for pandemic influenza

- Ensure emergency department representation at the Trust level.

- Talk about pandemic flu with your staff.

- Establish a process for fit testing all your staff.

- Institute a training programme for staff in the use of personal protection equipment (PPE).

- Consider stockpiling PPE.

- Agree the processes for managing potentially infected patients within the emergency department.

- Consider how roles in the hospital and within the emergency department may change during a pandemic.

- Establish a method for communicating with staff during a pandemic.

- Consider what you can do to improve staff morale.

- Consider and discuss the ethical decisions we will be required to make.

Health website ${ }^{2}$ and keep up to date with developments.

Identify staff concerns and aim to address them prior to the event. This is the time to calculate the percentage of your staff likely to be available for work during a pandemic. The current Department of Health guidance indicates that school closures are not a foregone conclusion, although recent work has suggested that prolonged school closure during a pandemic might reduce the cumulative number of cases and the peak attack rates. ${ }^{3}$ It is highly likely that either heads of schools will make the decision to close and have the authority to do so, or staff with some clinical knowledge will not be happy to continue to allow their children to attend school and will choose to take them out. Child care is clearly important but will be difficult to provide effectively and consistently during a pandemic. Ask staff to discuss with their families how they might make alternative arrangements for child care or elderly care. Many doctors in Hong Kong did not return home for a prolonged period (up to 3 months) to minimise any risk to their families. They either stayed in hospital accommodation or rented lodgings; our staff may need to consider if this is a possible option. If so, we need to estimate how much accommodation might be available on site or close to the hospital. Maximising the number of staff available to work is important, so ask them what would facilitate their ability to work.

\section{Establish a process for fit testing all your staff}

Health and Safety Executive (HSE) guidance ${ }^{4}$ recommends that masks are fit tested in advance to ensure they fit the wearer properly to provide maximum protection. One mask type does not fit everyone, so it is likely you will need to purchase additional types of masks for some of your staff. You may need to identify staff who are willing to be "fit testers", train them in the process of fit testing and have dedicated time in the week for staff to be fit tested. A central departmental register to record the type of mask each person can wear needs to be set up and kept up to date. The length of time it takes to do this should not be underestimated; it is a time-consuming process. To date we have fit tested 139 staff (49\% of the total) over a number of months; $23 \%$ of those tested have "failed" the test so we now need to source alternative masks. An alternative (and probably more realistic) approach is that the Trust should organise central fit testing for all staff as, in reality, all healthcare staff who come into contact with patients with flu will also require masks. Ensuring a mask will fit an individual properly is vital in assuring staff that their concerns regarding personal safety are being addressed.

\section{Institute a training programme for staff in the use of personal protection equipment (PPE)}

Although staff will be required to wear PPE during a pandemic, there are patients who are seen every day for whom we should probably be wearing PPE now. These patients include those from high-risk overseas areas, patients with possible open tuberculosis, and those with risk factors for avian influenza. One useful approach is to ask all patients about their "FTOCC" status $^{5}$ (see below).

Do not assume that staff (particularly medical staff) will be able to put on and remove PPE without contaminating themselves or others. All members of staff, including senior medical and nursing staff, will need to be trained to do this safely. In addition, you have a responsibility to ensure that all staff who work in the department are trained, so you will need to include both clinical and non-clinical staff such as cleaning and administrative staff, porters and other support staff.

Consider establishing a training team; make the training mandatory and record attendance and competence on the training database. The requirement for training may need to be incorporated into annual appraisals or job planning. Establish regular teaching and practice for staff in the use of PPE and infection control measures, and integrate these important aspects of training into induction procedures for new staff. Regular and appropriate training will help instil confidence in your staff, confidence they will need to call upon when coming to work will mean the certainty of exposure to a potentially lethal virus.

\section{Consider stockpiling PPE}

Procurement of PPE will potentially be difficult once a pandemic reaches the UK. Serious consideration should be given to stockpiling relevant PPE. There are obvious financial and stock rotation implications, but these can be minimised with some forward planning. Areas within hospitals that currently use FFP masks should be encouraged to move towards using FFP3 masks as a matter of routine. The yearly usage can then be calculated, purchased, held centrally and internally rotated as part of daily stock control. The benefit of introducing this system is that there will be stock immediately available for the initial wave of a pandemic and shelf life (currently 3 years) is not an issue.

Consideration should also be given to purchasing nondisposable FFP3 masks, enough to provide all staff working within the emergency department, infectious diseases wards and critical care areas, as this will provide a degree of staff protection in those areas that will be initially affected should disposable stocks become a problem. Knowledge of where stocks of surgical masks, gloves and gowns are kept, with approximate stock levels, should also be collated; these types of PPE can probably be redirected to acute clinical areas as elective services are curtailed. These agreements should be made now.

\section{Agree the processes for managing potentially infected patients} within the emergency department

We have to avoid unnecessary cross-infection within our departments. Assume every febrile patient could be infectious until you know otherwise. Consider how your department will 
screen for these febrile patients at the point of entry to the emergency department and subsequently isolate them from other non-febrile patients. In Hong Kong all patients are asked about the FTOCC criteria - those with Fever and answering yes to any one of the following four criteria are isolated and rapidly assessed. "TOCC" refers to any history of Travel to high-risk areas (such as Indonesia, certain provinces of China); Occupational history of exposure to chickens or other poultry (eg, chicken farmer); Contact history of exposure to other persons with febrile illness, particularly those with known disease; and Clustering (the presence of multiple ill patients arising from the same village, school, workplace or residence).

An IT programme that ensures up-to-date information about outbreaks of infectious diseases such as flu should be developed and available at triage so that high-risk areas are well known to triage staff.

In addition, non-invasive ventilation was not used in the SARS epidemic after it was found to increase aerosolisation of the virus and increase inter-patient infection. Nebulisers were also abandoned for the same reason. Consider updating staff in the use of metered dose inhalers and spacer devices for routine use rather than waiting until the epidemic arrives.

Think about the ventilation in your department and, in particular, identify if your department or your hospital has any negative pressure areas. It is important to work out how you will separate potentially infectious patients from non-infectious patients. In theory it is useful to separate febrile from nonfebrile patients, but bear in mind that not all febrile patients will have pandemic flu and not all patients who are afebrile will be disease-free. In the emergency department, cohorting may therefore be of questionable benefit.

However, the processes you decide upon need to practised and become embedded in everyday practice, as you will not know when the first infectious patient arrives at your department. While in the ideal world we would rebuild, this is not usually possible. If your emergency department is being refurbished or rebuilt, consider the importance of these measures during the planning process.

Think how your department might work after 50 days of a pandemic; save time and do it now. You will need to remember the need for gowning up and down areas-vital for the correct usage of PPE-and decide where these are best placed in your department. You should consider at what stage staff and visitors should be asked to wear surgical masks as a matter of routine. Review current plans in the light of new knowledge and liaise with colleagues in infection control and infectious disease.

\section{Consider how roles in the hospital and within the emergency department may change during a pandemic}

Identify which staff groups are most suitable to work in your department when their department stops routine work. You will need to define the core skills that will be required by redeployed staff working in the emergency department and develop a training and orientation programme for them. Liaison with the Trust is vital if time is to be available for this training. Consider holding a register of nurses who have previously worked in emergency departments who are aware that they are called on first to assist.

The pattern of attendances is likely to change during a pandemic, so staff may need to be reassigned within the department. The numbers of patients with minor injury or illness are likely to decrease significantly. Should this happen in Cambridge, we have decided to use some of our emergency nurse practitioners (ENPs) to act as "PPE patrols" with responsibility for ensuring staff put on their PPE correctly and remove it safely.

In Trusts which teach medical students, arrangements need to be made with the medical school involved in advance to ensure that student training can continue in some form or another if there is a major infectious diseases outbreak.

\section{Establish a method for communicating with staff during a pandemic}

Discuss what information staff would wish to receive: this may be the numbers of patients admitted with flu, the progress of sick colleagues or available supplies of PPE. Assign this role to a senior clinician or manager who is able to produce this information on a daily basis.

Remember that the Department of Health will issue communications advising on the standards of care and expectations of care in the event of a pandemic. This information must be incorporated into staff briefings and staff should be involved in local discussions about care provision.

Communication must be two-way during a flu pandemicwhile staff are always keen to receive up-to-date information, they must also have an easily accessible route to feed information and opinions back to the emergency department and hospital and Trust level management. This feedback must be responded to in a timely manner and any staff concerns addressed.

It may be necessary to provide information to staff as well as patients via the mass media. Normal press liaison procedures should be used as much as possible, but it may be prudent to have a secondary plan for media alerts for pandemic flu as regular press officers may also be unwell or absent from work due to domestic commitments.

\section{Consider what you can do to improve staff morale}

Morale is likely to be poor among staff working in a high-risk environment for long hours without respite and away from their families. Ask staff what would help improve their morale in this situation and give serious consideration to implementing these in the event of a pandemic. Suggestions from our staff range from the availability of healthy food throughout the day, free coffee and snacks, laundry facilities to free telephone calls and the availability of Skype or Google Talk.

\section{Consider and discuss the ethical decisions we will be required to make}

Ethical considerations are particularly relevant to emergency medicine which will have to cope with the increased numbers of critically ill patients with limited facilities. The Department of Health's ethical framework document ${ }^{6}$ provides a useful summary of the relevant ethical principles and offers some helpful advice but, in reality, it provides little practical help for those clinicians who will be asked to make decisions regarding treatment.

It will not be possible to admit all patients with flu during a pandemic outbreak. Difficult decisions will need to be made. Advice sheets may be prepared in advance to be distributed to patients and to caregivers at home. This should include advice on avoidance of cross-infection, symptoms which should lead to a further consultation with medical services (this advice may vary depending on the level of pandemic outbreak) and details of the likely course of the illness. 


\section{Box 2 Glossary of terms}

\section{UK Alert level 1}

This equates to the World Health Organization phase 6, where there is increased and sustained transmission in the general population (ie, a pandemic is confirmed). UK Alert level 1 means there are reported cases outside the UK only.

\section{FFP3}

A filtering face piece device (ie, a mask certified to the PPE directive).

\section{Category 1 responders}

An NHS Trust established under section 5 of the National Health Service and Community Care Act 1990 or a Foundation Trust established under section 1 of the Health and Social Care Act 2003 which has the function of providing hospital accommodation and services in relation to accident and emergency.

\section{Fit testers}

FFP3 masks need to be fitted with care to ensure they fit as well as possible, especially around the nose and mouth. Fit testers are staff who are specifically trained to fit masks and test the fit to ensure it is adequate for personal safety.

\section{FTOCC}

Five screening questions used to identify those at risk of infection: history of Fever, Travel to high-risk areas, Occupational history, Contact history and Clustering of cases.

It is likely that there will be a significant number of appropriate patients who will suffer potentially avoidable deaths because the ventilatory support or critical care they require is not available. It is particularly difficult to envisage how the principle of fairness outlined in the Department of
Health document will be achievable in such circumstances. We have no answers to such ethical dilemmas; all we can do is warn our staff of the difficult choices we will have to face should a pandemic occur.

\section{CONCLUSION}

Not preparing for a pandemic may mean some of your staff may fall ill or even die because of unnecessary exposure to a virulent virus. It could result in a level of staff absenteeism that is so high that patients die for want of treatment. We believe these practical suggestions may help you to maximise your preparation for your staff for what may lie ahead.

Acknowledgements: We would like to thank our colleagues in Hong Kong for sharing their experiences with us. These were Professor T H Rainer, Mr Stones Wong, Professor C D Gomersall, Professor G M Joynt and Professor N Lee of the Prince of Wales Hospital and Miss Wu of the Princess Margaret Hospital. We also thank Mr Jim Wardrope for his encouragement to publish.

Competing interests: None.

\section{REFERENCES}

1. Department of Health. Pandemic flu: surge capacity and prioritisation in health services - provisional UK guidance. London: Department of Health, February 2008. http://www.dh.gov.uk/en/Publicationsandstatistics/Publications/ PublicationsPolicyAndGuidance/DH 080744 (accessed 15 April 2008).

2. Department of Health. Pandemic flu. London: Department of Health. http://www.dh gov.uk/en/Publichealth/Flu/PandemicFlu/index.htm (accessed 15 April 2008).

3. Cauchemez S, Valleron AJ, Boelle PY, et al. Estimating the impact of school closure on influenza: transmission from sentinel data. Nature 2008;452:750-4.

4. Health and Safety Executive. Pandemic flu: workplace guidance. London: Health and Safety Executive, February 2008. http://www.hse.gov.uk/biosafety/diseases/ pandemic.htm (accessed 15 April 2008).

5. Illingworth RN. General approach - infection control and prevention. In: Wyatt JP, Illingworth RN, Graham CA, et al, eds. Oxford handbook of emergency medicine. 3rd ed. Oxford: Oxford University Press, 2006:34-5.

6. Department of Health. Responding to pandemic influenza: the ethical framework for policy and planning. London: Department of Health, November 2007. http://www.dh. gov.uk/en/Publicationsandstatistics/Publications/PublicationsPolicyAndGuidance/ DH_080751 (accessed 15 April 2008). 